Check for updates

Kent

Cite this as: BMJ 2022;376:0222 http://dx.doi.org/10.1136/bmj.0222 Published: 26 January 2022

\title{
Covid-19: One in 23 people in England had infection in early January
}

\section{Jacqui Wise}

Coronavirus infections in England were at their highest ever rate in early January this year, with an estimated one in 23 people infected, the ongoing REACT-1 monitoring study has reported. ${ }^{1}$

Findings from Imperial College London and Ipsos Mori covering 5-20 January show that the prevalence in England was $4.41 \%$. This is more than three times the prevalence in the previous round of the survey in December, when one in 70 had the virus.

Infections reached a peak around 5 January before levelling off from mid-January, but they were still at extremely high levels. Schoolchildren have shown a rise in prevalence after returning to school this month.

The study authors said that they observed "unprecedented" levels of infection with SARS-CoV-2 in England in January 2022, with almost complete replacement of the delta variant by omicron. They warned that the increase in the prevalence of infection in children could pose a risk to adults despite the current decline among adults.

\section{Age and region}

For round 17 of the study 100607 people swabbed themselves at home, and 4011 samples showed positive when analysed by PCR. The highest weighted prevalence was seen in children aged 5-11 (7.81\%), and the next highest was in 12-17 year olds. Over $75 \mathrm{~s}$ had the lowest rate at $2.43 \%$, although this was still a near 12-fold increase from December.

Prevalence increased in every region when compared with the previous round of the study, with the highest figure recorded in the north east $(6.85 \%)$, followed by Yorkshire and the Humber (5.58\%). Of 1406 samples sequenced, $99 \%$ were the omicron variant. Risks of infection were higher among key workers, people living in large households, and those in the most deprived areas.

Among 3582 people who tested positive, almost two thirds $(64.6 \%)$ reported a confirmed previous infection and $7.5 \%$ reported a suspected previous infection. Currently, the daily figures of covid cases reported by the government do not include reinfections; however, this will change on 31 January, when anyone in England who has a second positive test more than 90 days after the first will be added to the official figures.

Paul Elliott, director of the REACT programme from Imperial College's School of Public Health, said, "There is good news in our data in that infections had been rapidly dropping during January, but they are still extremely high and may have recently stalled at a very high prevalence.

"Of particular concern is that there is rapidly increasing prevalence among children now they are mixing more following the start of the school term and, compared with December, prevalence in older people aged 65 plus has increased seven- to 12-fold, which may lead to increased hospitalisations."

The researchers also looked at existing publicly available data and found that, although trends on hospital admissions and deaths continued to track infections, this was at a lower level than before the widespread rollout of the vaccination campaign. However, they said that continued monitoring would be important over the coming weeks in view of the continued high levels of infection, including among the older population, and as restrictions are lifted.

1 Elliott P, Eales O, Bodinier B, et al. Post-peak dynamics of a national Omicron SARS-CoV-2 epidemic during January 2022. Imperial College London. 26 Jan 2022. https://spiral.imperial.ac.uk/handle/10044/1/93887

This article is made freely available for personal use in accordance with BMJ's website terms and conditions for the duration of the covid-19 pandemic or until otherwise determined by BMJ. You may download and print the article for any lawful, non-commercial purpose (including text and data mining) provided that all copyright notices and trade marks are retained. 